\title{
Comparative transcriptomic analyses revealed divergences of two agriculturally important aphid species
}

Dahai Wang ${ }^{1,2}$, Qi Liu ${ }^{3}$, Huw D Jones ${ }^{4}$, Toby Bruce ${ }^{4}$ and Lanqin Xia ${ }^{1 *}$

\begin{abstract}
Background: Grain aphid (Sitobion avenae F) and pea aphid (Acyrthosiphon pisum) are two agriculturally important pest species, which cause significant yield losses to crop plants each year by inflicting damage both through the direct effects of feeding and by vectoring debilitating plant viruses. Although a close phylogenetic relationship between grain aphid and pea aphid was proposed, the biological variations between these two aphid species are obvious. While the host ranges of grain aphid is restricted to cereal crops and in particular wheat, that of pea aphid is wider, mainly colonizing leguminous plant species. Until now, the genetic factors underlying the divergence between grain aphid and pea aphid still remain unclear due to the limited genomic data of grain aphid available in public databases.

Results: Based on a set of transcriptome data of grain aphid generated by using Roche 454 GS-FLX pyrosequencing, comparative analysis between this set of transcriptome data of grain aphid and mRNA sequences of pea aphid available in the public databases was performed. Compared with mRNA sequences of pea aphid, 4,857 unigenes were found to be specifically presented in the transcriptome of grain aphid under the rearing conditions described in this study. Furthermore, 3,368 orthologous pairs which could be calculated with both nonsynonymous (Ka) and synonymous (Ks) substitutions were used to infer their sequence divergences. The average differences in the coding, $5^{\prime}$ and $3^{\prime}$ untranslated regions of these orthologs were 10.53\%, 21.29\% and 18.96\%, respectively. Moreover, of 340 orthologs which were identified to have evolved in response to positive selection based on the rates of Ka and Ks substitutions, 186 were predicted to be involved in secondary metabolism and xenobiotic metabolisms which might contribute to the divergence of these two aphid species.

Conclusions: The comprehensive transcriptome divergent sequence analysis between grain aphid and pea aphid provides an invaluable resource for the investigation of genes involved in host plant adaptation and evolution. Moreover, the demonstration of divergent transcriptome sequences between grain aphid and pea aphid pave the way for the investigation of the molecular mechanisms underpinning the biological variations of these two agriculturally important aphid species.
\end{abstract}

Keywords: Grain aphid, Pea aphid, Transcriptome, Comparative transcriptomic analysis, Divergence

\footnotetext{
* Correspondence: xialanqin@caas.cn

${ }^{1}$ Institute of Crop Sciences /The National Key Facility for Crop Gene Resource and Genetic Improvement, Chinese Academy of Agricultural Sciences (CAAS), 12 Zhongguanchun South Street, Beijing 10081, China

Full list of author information is available at the end of the article
} 


\section{Background}

Aphids are major agricultural pests which cause significant yield losses to crop plants each year by inflicting damage both through the direct effects of feeding and by vectoring debilitating plant viruses [1]. Annual worldwide crop losses due to aphids are estimated at hundreds of millions of dollars [2]. The application of nitrogen fertilizer and elevation of atmospheric $\mathrm{CO}_{2}$ concentration exacerbate aphid infestation [3]. The major aphid species infesting wheat in China are the grain aphid (Sitobion avenae F.), greenbug (Schizaphis graminum Rondani), bird-cherry oat aphid (Rhopalosiphum padi Linnaeus) and rose-grain aphid (Metopolophium dirhodum Walker). Of these, grain aphid is the most dominant and destructive one, affecting most of the wheat production areas [4]. The grain aphid is also a major pest of wheat in Europe and North America [5]. In some wheat production areas, it has damaging infestations every year, causing the wide-spread use of chemicals and as much as 15 to $60 \%$ of severe reduction in wheat yield [6]. Due to the complexity of plant-aphid interactions and the rapid development of resistant pest biotypes, outbreak of aphids causing substantial losses of wheat are reported regularly [5,6].

Aphids are important agricultural pests and also biological models for study of insect-plant interactions, virus vectoring and host plant adaptation [1]. Recently, the release of genome sequence of the pea aphid provided a foundation for post-genomic studies of fundamental biological questions both in pea aphid and other aphid species. It revealed the presence of more coding genes than in previously sequenced insects and in particular, the presence of genes with no orthologs in other insects [1]. Now, the assembled genome sequence data, ESTs and full length cDNAs of the pea aphid are accessible at the AphidBase web portal (www.aphidbase.com) [7]. With the release of the first aphid genome and the general accessibility of next generation sequencing technologies, there is an expectation within the aphid research community that we have reached a tipping point for genome information from additional aphid species and high-resolution comparative genomic and evolutionary analyses [8]. Although a close phylogenetic relationship between pea aphid and grain aphid was illustrated based on analysis of partial sequence of EF1 alpha orthologs in different aphid species [8], the diversity between these two aphid species is obvious. For example, the host ranges of grain aphid are restricted to cereal crops and in particular wheat, that of pea aphid is wider, mainly colonizing many leguminous plant species. Until now, the molecular mechanisms underlying their divergence and host adaptation have not been documented due to the lack of enough genetic data of grain aphid available in the public databases. In a previous study, we performed de novo transcriptome assembly and gene expression analyses of the alimentary canals of grain aphids before and after feeding on wheat plants by using Illumina RNA sequencing [9]. However, a complete transcriptome and/or genomic data of the grain aphid have not been documented so far, in spite of the fact that grain aphid is most dominant and destructive aphid pest affecting wheat production in China, Europe and North America.

Next-generation sequencing (NGS) technologies allow direct sequencing of cDNA generated from messenger RNA (RNA-seq) [10]. These new technologies enable the de novo reconstruction of the transcriptome for a non-model organism [11], leading to novel opportunities for expression profiling of organisms lacking any genome or transcriptome sequence information [11,12]. In this paper, in order to reveal the genetic factors underlying the divergence between the grain and pea aphids, comparative analysis between the transcriptome data of grain aphid generated in our lab by Roche 454 GS-FLX pyrosequencing and mRNA sequences of pea aphid available in the public databases was performed. A number of orthologous genes that showed signs of diversifying natural selection were identified. This comparative analysis provides an invaluable resource for the investigation of genes involved in plant infestation, host adaptation and insecticide resistance. Moreover, the demonstration of divergent transcriptome sequences between grain aphid and pea aphid pave the way for the investigation of the molecular mechanisms underpinning the biological variations of these two destructive aphid species.

\section{Results}

Roche 454 GS-FLX pyrosequencing and assembly of grain aphid transcriptome

To obtain a global view of the grain aphid transcriptome, 300 grain aphids at different developmental stages, which were derived from a single clonal lineage, were used for RNA isolation. High-throughput RNA sequencing (RNAseq) was performed with Roche 454 GS-FLX pyrosequencing platform. A total of 1,106,696 reads, with an average length of $380 \mathrm{bp}$, amounting to a total $401.7 \mathrm{Mb}$, were obtained and deposited in the NCBI Short Read Archive (SRA) under the accession number: SRA065628. The raw reads were assembled using Mira 3 assembly software packages (www.chevreux.org/projects_mira.html), and resulted in 44,682 contigs with an average length of 812.5 bp (Table 1). The un-assembled high quality reads which occupied $1.79 \%(16,677)$ of the total cleaned reads were added to the contigs dataset and generated in total of 61,359 sequences. These sequences were further assembled with gsAssembler v 2.3 software. Among 61,359 sequences, 29,679 were identified as unique sequences, while the remaining 31,680 sequences were re-assembled into 3,513 
Table 1 Summary of the transcriptome of grain aphid

\begin{tabular}{ll}
\hline Items & Values \\
\hline Total number of reads (bp) & $1,106,696$ \\
Total base pairs (Mb) & 401.7 \\
Average read length (bp) & 380 \\
Total number of contigs & 31,199 \\
Singletons & 1,078 \\
Total unigenes & 32,277 \\
N50 of contigs (bp) & 1,021 \\
N90 of contigs (bp) & 479 \\
Average length of singletons (bp) & 369 \\
The longest length of contigs (bp) & 11206 \\
Average length of contigs (bp) & 866 \\
Average length of unigenes (bp) & 850 \\
\hline
\end{tabular}

contigs. After removing sequences below 100 bp and transcripts of Buchnera aphidicola (an endosymbiont of aphids), we obtained 32,277 distinct sequences as unigenes which included 31,199 contigs and 1078 singletons (Table 1). The flow chart of data assembly was provided in the Additional file 1. The assembled transcriptome data were submitted and have been deposited at DDBJ/EMBL/ GenBank under the accession GAPL00000000. The average coverage of the contigs was $15.5 \mathrm{x}$ (which was calculated by dividing total length of assembled reads by length of all contigs), and the longest length and the average length of the assembled contigs were 11,206 bp and $866 \mathrm{bp}$, respectively (Table 1 ). Next, we analyzed the length distribution of the contigs, singletons, unigenes and the number of reads per unigene. As shown in Figure 1, 7,878 contigs $(25.3 \%)$ were longer than $1000 \mathrm{bp}, 14,362$ contigs (46.0\%) ranged from $500 \mathrm{bp}$ to $1000 \mathrm{bp}$, whereas 8946 contigs $(28.7 \%)$ were below 500 bp (Figure 1). The N50 and N90 of the contigs, were 1,021 and $479 \mathrm{bp}$, respectively (Table 1 ). The un-assembled high quality reads were assigned as singletons, which occupied $0.12 \%(1,078)$ of the total cleaned reads. The length of singletons varied from $100 \mathrm{bp}$ to $600 \mathrm{bp}$ with an average of $369 \mathrm{bp}$. The majority of singletons were between $200 \mathrm{bp}$ and $600 \mathrm{bp}$ in length, and $10.3 \%$ of them were below 200 bp (Figure 1). The number of reads per unigene varied from 1 to 13,917 with an average of 32.5 . The group that consisted of less than 5 reads contained 16,674 unigenes, representing $51.7 \%$ of the total assembled unigenes. Whereas 117 unigenes had more than 1000 reads, 175 unigenes had 501 to 1000 reads, and 1,396 unigenes had 100 to 500 reads. Unigenes with reads below 100 represented $94.7 \%(30,588)$ of the total number of unigenes (Figure 1).

The A, T, C and $\mathrm{G}$ proportions of the assembled unigenes were $30.02 \%, 30.42 \%, 19.81 \%$ and $19.64 \%$, respectively. The GC content of the transcriptome data of grain aphid was $39.45 \%$, a value slightly higher than that of the pea aphid which was $38.8 \%$ [1]. Of these 32,277 unigenes assembled, the average length of coding regions (CDSs) was $556 \mathrm{bp}$ and the GC content of the CDSs was 42.39\%. Among which, 17,256 unigenes, of which GC content was $31.73 \%$, could be predicted with 3'UTRs and the average length of the 3'UTRs was $282.6 \mathrm{bp}$. At the same time, 16,846 unigenes were predicted with 5' UTRs, of which GC content was $36.42 \%$ and the average length of 5'UTRs was $262.3 \mathrm{bp}$. It was interesting to note that the GC contents among CDSs, 5'UTRs and 3' UTRs were different and the CDSs had highest GC content among these three categories, whereas the GC content of 5 'UTRs was $4.69 \%$ higher than that of 3 'UTRs.

\section{The functional annotation and classification of the assembled unigenes}

Functional annotations of the derived unigenes were performed using Basic Local Alignment Search Tool (BLAST) against the public available databases such as National Center for Biotechnology Information (NCBI) Nucleotide Acid Database (Nt) and Non-redundancy Protein Database $(\mathrm{Nr})$, and BLASTx against the Swiss-Prot Database. A total of 25,389 unigenes $(78.7 \%)$ matched the $\mathrm{Nt}$ database with an $\mathrm{E}$ value of $1 \mathrm{E}-10$, and 21,635 unigenes (67.9\%) matched genes in $\mathrm{Nr}$ database with $\mathrm{E}$ value of 1E-5. BLASTx against Swiss-Prot Database indicated that 16,211 unigenes $(50.2 \%)$ were annotated with an E-value of $1 \mathrm{E}-5$ (Table 2, See Additional file 2).

All unigenes that matched Swiss-Prot database entries were further classified by Gene Ontology (GO) terms according to the number of the matched entries. In total, 11,731 unigenes (36.3\%) had GO terms (Table 2, See Additional file 2). Furthermore, the unigenes were also classified according to Eukaryotic Orthologous Groups (KOG) terms. In total, 15,957 unigenes (49.4\%) had KOG terms (Table 2, See Additional file 2). In addition, 13,876 unigenes (43\%) were mapped to the Kyoto Encyclopedia of Genes and Genomes (KEGG) pathways (Table 2) and grouped into 193 categories. The majority of classifications were metabolic pathways and biosynthesis of secondary metablolites, which represented $19.15 \%$ and $6.05 \%$ of total annotated unigenes, respectively.

\section{The transcriptomic divergences between grain aphid and pea aphid}

Comparisons of the transcriptome data of different aphid species would provide useful information in deciphering many of the specialized biological variations underlying the role of aphids as plant pests, and in understanding the transcriptome evolution and the genetic factors underlying the divergence of these species. To compare the sequence divergence of these two aphid species, we analyzed the possible orthologous genes between the transcriptome of grain aphid obtained in this study and the pea aphid 


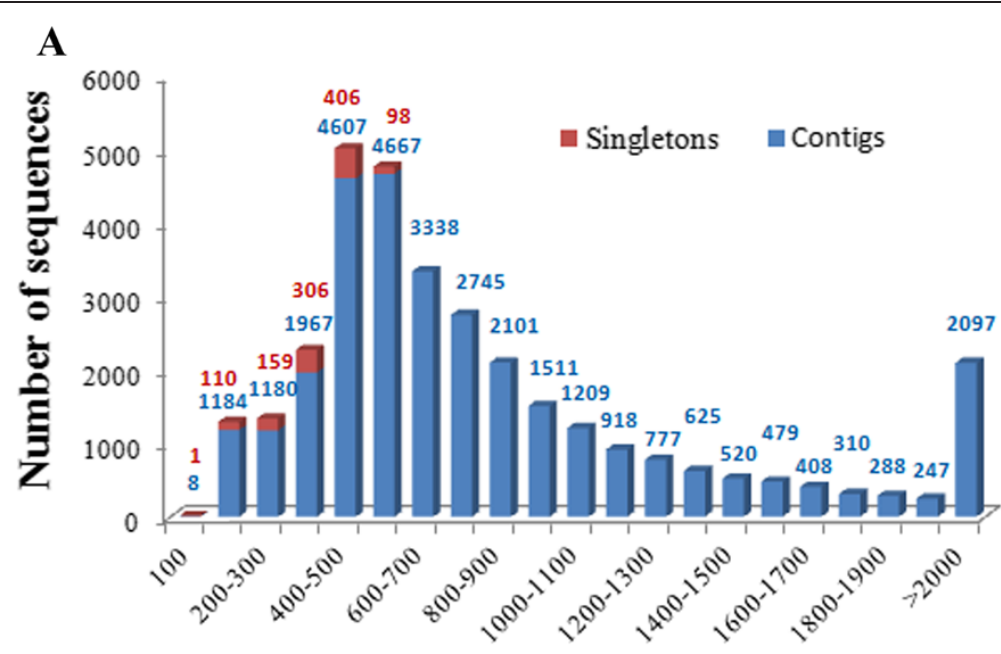

\section{Length of distribution (bp)}

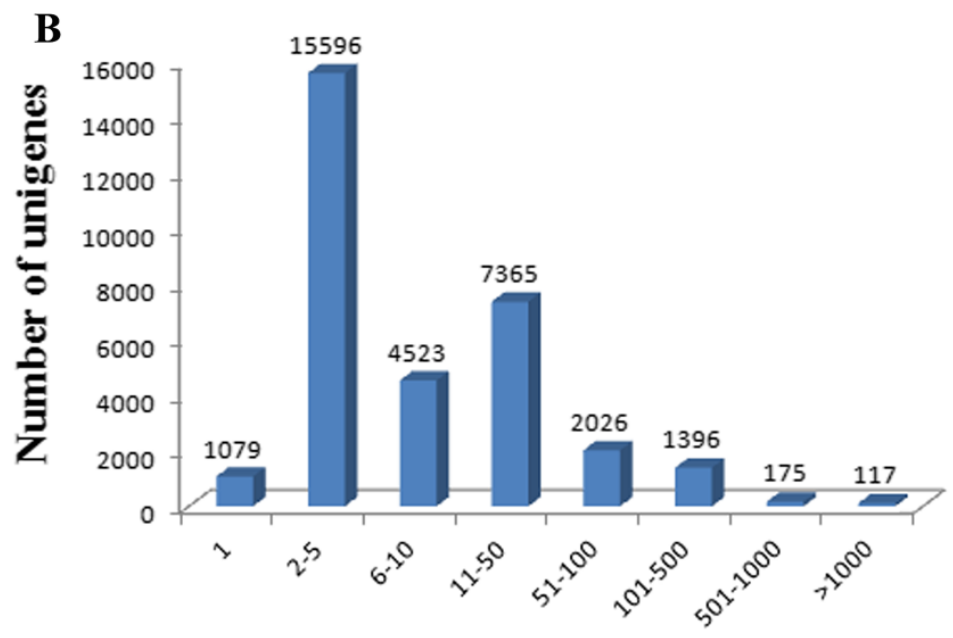

Number of reads

Figure 1 Length distribution of contigs, singletons and unigenes and the distribution of reads number of the assembled unigenes.

(A) The length distributions of contigs, singletons and unigenes. (B) The reads distribution of unigenes.

Table 2 Numbers of annotated unigenes in the public databases

\begin{tabular}{lll}
\hline Annotation databases & $\begin{array}{l}\text { Annotated } \\
\text { unigene number }\end{array}$ & Percentage (\%) \\
\hline $\mathrm{Nt}$ & 25,389 & 78.7 \\
$\mathrm{Nr}$ & 21,635 & 67.0 \\
Swiss-Prot & 16,211 & 50.2 \\
KEGG & 13,876 & 43.0 \\
GO & 11,731 & 36.3 \\
KOG & 15,957 & 49.4 \\
Total assembled unigenes & 32,277 & \\
\hline
\end{tabular}

mRNA sequence data which are available in website (https://www.aphidbase.com/aphidbase/content/download/ 3250/33670/file/aphidbase_2.1b_mRNA.fasta.bz2) using bidirectional best hit which has been widely used to identify orthologous genes [13,14].

First, BLASTn and tBLASTx tools were employed to screen out unigenes homologous to pea aphid. Using BLASTn tool, 25,294 unignes homologous to pea aphid mRNA sequences were identified and 6,983 sequences un-matched were further subjected to comparison with pea aphid mRNA data at translation level. As sequences below 250 bp were too short to be translated or compared 
with the translated sequences from pea aphid mRNA, they were removed from the 6,983 sequences dataset, and finally 6,230 sequences remained. Furthermore, by using tBLASTx tool, we found that among 6,230 sequences, 1,114 sequences were orthologous to pea aphid mRNA sequences. For the remaining 5,116 sequences, 259 were annotated with predicted functions similar to these of sequences from pea aphid in Nt database. Thus, among the 32,277 unigenes assembled, 26,667 unigenes $(25,294+$ $1,114+259$ ) which occupied $81.8 \%$ of total assembled unigenes showed homology to pea aphid mRNA sequences. Of these, 15,444 were one-to-one orthologs, 2,170 were tree-matched orthologs, and the remaining 9,053 were either homologous to pea aphid mRNA sequences with the ratio of matched region below $50 \%$ or some paralogs (Figure 2). During BLASTn analysis, 753 sequences which were shorter than $250 \mathrm{bp}$ and had no homologous sequences with pea aphid mRNA identified by BLASTn tool were excluded because they were too short for tBLASTx searching. The remaining 4,857 unigenes (32,277-26,667753 ) were identified to be grain aphid specific genes under the described rearing conditions in this study (Figure 2). The presence and expression of some of the grain aphid specific genes were confirmed by qRT-PCR with randomly selected 14 unigenes (Data not shown).

Furthermore, the Nt, Nr, Swiss-Prot annotations and KEGG, KOG and GO classifications of 4,857 grain aphid specific unigenes which had no homologous sequences with pea aphid were performed. As indicated in Table 3, less than $15 \%$ of these unigenes were predicted with a defined function, whereas the rest of them could not be annotated or classified in the current available public databases, indicating that the functions of these sequences remain unknown and need to be investigated or annotated in the future. In addition, The KEGG classification result showed that among 4,857 grain aphid specific unigenes, 607 unigenes was annotated with KEGG terms which most of them were belonged to the groups of metabolic pathways (33.9\%), biosynthesis of secondary metabolites (13.3\%), protein processing in endoplasmic reticulum (4.3\%), purine metabolism (4.0\%), oxidative phosphorylation (3.5\%), starch and sucrose metabolism (3.1\%), aminobenzoate degradation (2.8\%), naphthalene degradation (2.5\%), ploycyclic aromatic hydrocarbon degradation $(2.3 \%)$, bisphenol degradation $(2.0 \%)$, chlorocyclohexane and chlorobenzene degradation $(2.0 \%)$ and so forth (Figure 3, See Additional file 3). Given the differences of the host plants and the habitats of these two aphid species, we proposed that the above mentioned pathways might play an important role in biological variations and divergences between pea aphid and grain aphid.

\section{The sequence divergences of orthologs between grain and pea aphids}

Except for the identified distinct grain aphid and pea aphid specific sequences, the sequence divergences inside the orthologous gene pairs between grain aphid and pea aphids were also estimated. Among 26,667 unigenes homologous to pea aphid mRNA sequences, in total 17,614 sequences pairs were assigned as orthologs. For

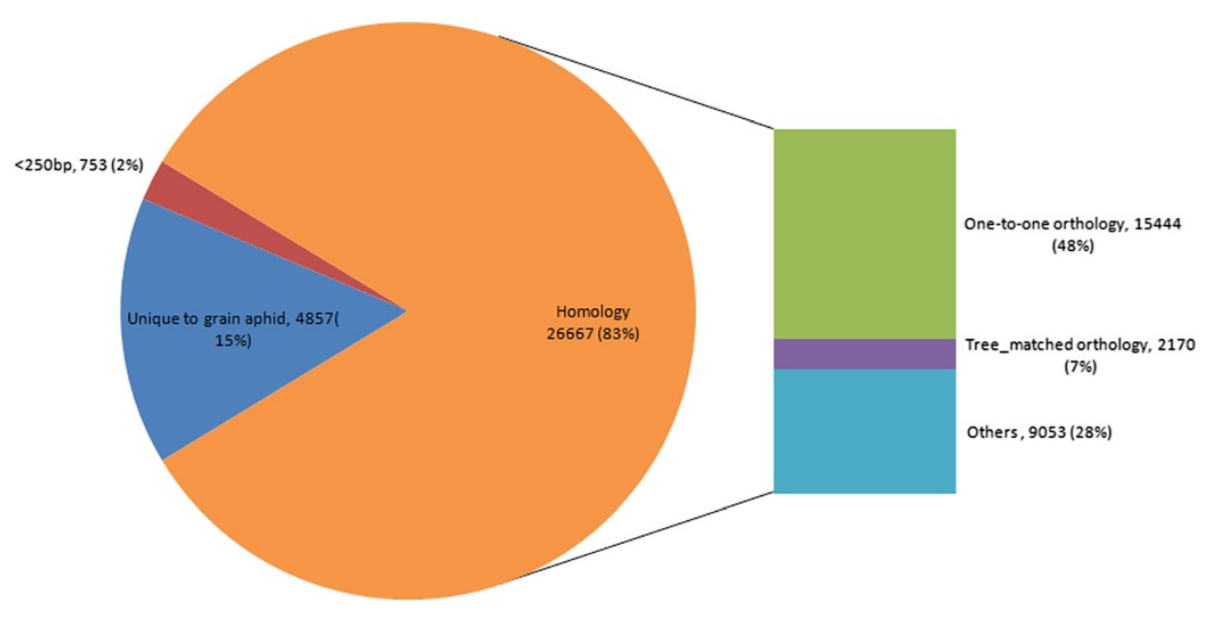

Figure 2 Comparison of the assembled grain aphid unigenes with pea aphid mRNA sequences. Among the 32,277 unigenes assembled, 26,667 grain aphid unigenes (yellow) were homologous to pea aphid mRNA sequences, of these, 15,444 were one-to-one orthologs (green), 2,170 were tree-matched orthologs (purple), and 9,053 were homologous to pea aphid mRNA sequences with the ratio of matched region below $50 \%$ and some paralogs (light blue). The number of unigenes which were unique to grain aphids compared with pea aphid mRNA was 4,857 (blue). And the remaining 753 sequences (red) which were shorter than 250 bp and had no homologous sequences with pea aphid mRNA identified by BLASTn tool were excluded because they were too short sequence for tBLASTx searching. All orthologs were identified using ETE pipeline with SOS of 0.0 . 
Table 3 Annotations of the 4,857 grain aphid specific unigenes which had no orthologs in pea aphid

\begin{tabular}{lll}
\hline $\begin{array}{l}\text { Public databases } \\
\text { for annotation }\end{array}$ & Annotated unigenes & Percentage (\%) \\
\hline $\mathrm{Nt}$ & 205 & 4.2 \\
$\mathrm{Nr}$ & 442 & 9.1 \\
Swiss-Prot & 722 & 14.9 \\
GO & 161 & 3.3 \\
KOG & 530 & 10.9 \\
KEGG & 607 & 12.5 \\
\hline
\end{tabular}

the 17,614 orthologous gene pairs identified between grain aphid and pea aphid, the CDS of each unigene was obtained by removing $5^{\prime}$ untranslated regions (UTRs) and 3' UTRs sequence and aligned separately to its counterpart mRNA sequence of pea aphid using a MegaBlast algorithm to generate matched orthologous pairs. The errors caused in the orthologous pair alignment were checked and removed manually. Then, the orthologous pairs below 150 base pairs were also removed in order to generate reasonable results. Finally, 4,191 pairs with CDS larger than 150 base pairs obtained and subjected to KaKs Calculator analysis [15], of these, only 3,368 orthologous pairs could be calculated with non-synonymous (Ka), synonymous (Ks) and their substitution ratio (Ka/Ks).

The sequence divergences within 3,368 orthologous pairs between the two aphid species, of which ratios $\mathrm{Ka} /$ Ks substitution could be calculated, had been assessed. For the 5'UTR, the GC content was $38.29 \%$ and $4.93 \%$ of the compared nucleotides were in a CpG contexts (Table 4). Differences between 5'UTRs of the analyzed orthologous genes of grain aphid and pea aphid occurred at $21.29 \%$ of the positions. Interestingly, $\mathrm{CpG}$ sites in the 5 'UTR differed at $10.94 \%$ of positions, whereas nonCpG sites differed at $21.31 \%$. Thus, within 5'UTRs, differences occurred approximately 2 times more often at non-CpG sites than at CpG sites. For the 3'UTR, the GC content was $26.90 \%$ and $1.04 \%$ of the nucleotides was in a CpG context. The overall difference of 3'UTR between these two aphid species was $18.96 \%$. CpG and non-CpG sites differed at $32.43 \%$ and $18.93 \%$, respectively. Hence, in the 3'UTR, CpG sites contained more differences than non-CpG sites. To understand the mechanism of evolution, we compared the ratio of transition (ts) and transversion (tv) $[16,17]$. Overall, the transitional differences were more frequent than transversional differences in 5'UTRs and 3' UTRs (Table 4). Interestingly, the ts/tv

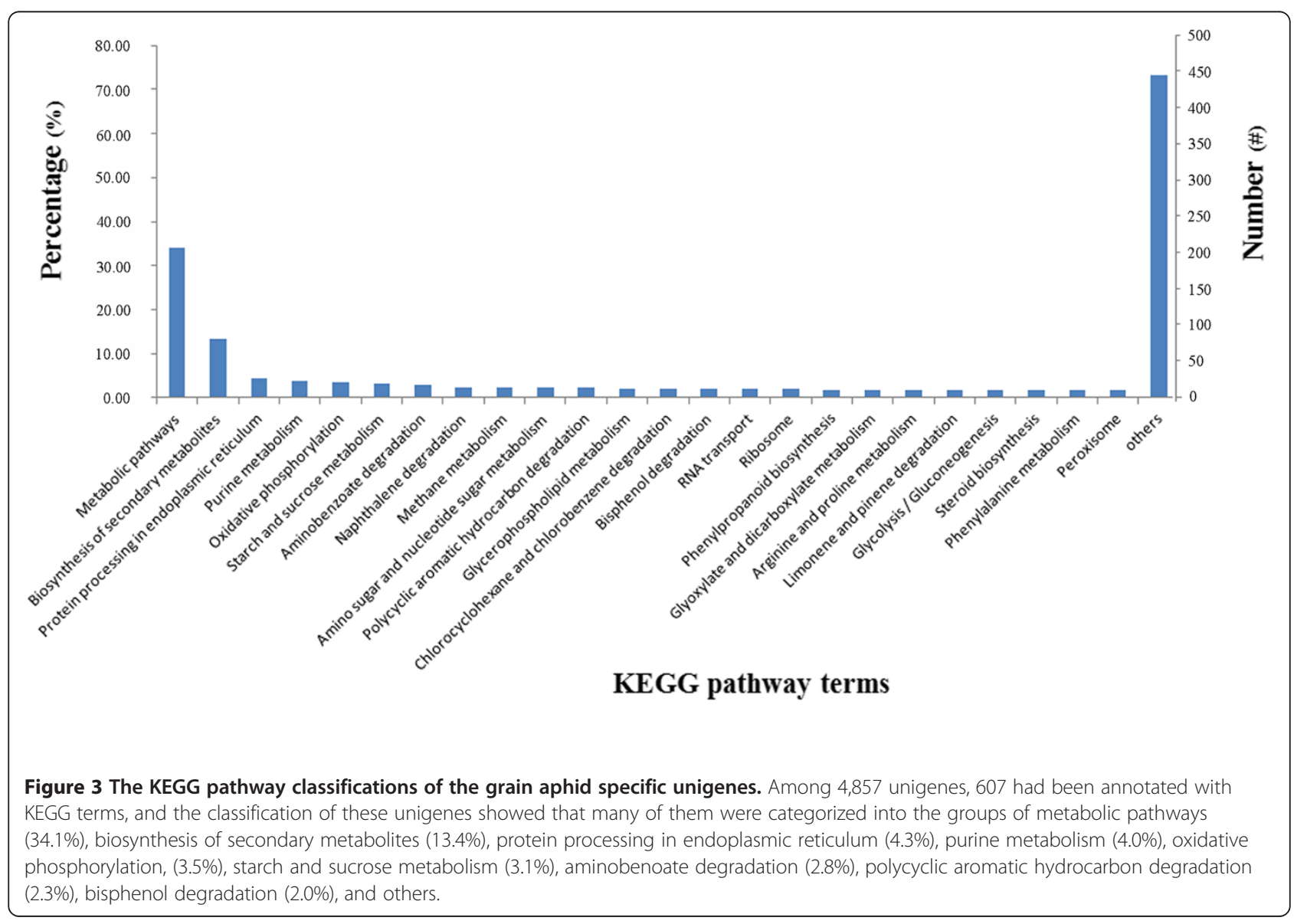


Table 4 Sequence divergences within orthologous gene pairs between the grain aphid and pea aphid

\begin{tabular}{|c|c|c|c|c|c|c|}
\hline \multirow[t]{3}{*}{ 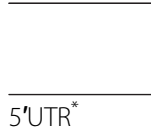 } & \multirow[t]{2}{*}{$\%$ CpG } & \multirow[t]{2}{*}{$\% \mathrm{GC}$} & \multicolumn{2}{|c|}{ Differences (\%) } & \multirow[t]{2}{*}{ Compared (kb) } & \multirow[t]{2}{*}{$\mathrm{ts} / \mathrm{tv}^{* * * * *}$} \\
\hline & & & Mean & SE & & \\
\hline & 4.93 & 38.29 & & & & \\
\hline ALL & & & 21.29 & 15.44 & 638.45 & 1.14 \\
\hline Non CpG & & & 21.31 & 15.42 & 606.96 & 1.14 \\
\hline CpG & & & 10.94 & 13.71 & 31.49 & 1.35 \\
\hline $\operatorname{CDS}^{* *}$ & 15.61 & 41.47 & & & & \\
\hline ALL & & & 10.53 & 15.56 & 4713.66 & 0.97 \\
\hline Non CpG & & & 11.37 & 16.61 & 3977.8 & 1.00 \\
\hline CpG & . & & 13.32 & 19.93 & 735.86 & 0.89 \\
\hline nd sites $^{* * *}$ & 15.71 & 45.04 & & & & \\
\hline ALL & & & 8.30 & 15.30 & 3009.53 & 0.72 \\
\hline Non CpG & & & 9.10 & 16.24 & 2536.70 & 0.74 \\
\hline CpG & & & 10.74 & 19.78 & 472.83 & 0.65 \\
\hline $4 d$ sites $^{* * * *}$ & 19.88 & 37.20 & & & & \\
\hline ALL & & & 16.08 & 16.72 & 652.40 & 1.03 \\
\hline Non CpG & & & 17.03 & 18.23 & 522.67 & 1.03 \\
\hline CpG & & & 20.25 & 20.67 & 129.73 & 1.03 \\
\hline $3^{\prime} U T R$ & 1.04 & 26.90 & & & & \\
\hline ALL & & & 18.96 & 14.53 & 905.70 & 1.12 \\
\hline non $\mathrm{CpG}$ & & & 18.93 & 14.49 & 896.27 & 1.12 \\
\hline $\mathrm{CpG}$ & & & 32.43 & 19.81 & 9.43 & 1.46 \\
\hline
\end{tabular}

* UTRs: untranslated regions.

**CDS: coding sequence.

***nd sites: non-degenerative sites.

****4d sites where no changes cause any amino acid replacement.

*****ratio of transitions (ts) over transversions (tv)

Note: The total loci of orthologous gene pairs examined between these two aphid species is 3368 .

ratio was higher in the $\mathrm{CpG}$ positions ( 1.40) than the nonCpG positions ( 1.10) in both the 5'UTRs and 3' UTRs. When comparing divergences of $3^{\prime}$ UTR and 5'UTR, the CPG sites divergence of 3'UTR (1.46) was higher than that of 5'UTR (1.35); however, the overall and non-CpG sites divergence of 5 'UTR $(1.14,1.14)$ was higher than that of 3'UTR $(1.12,1.12)$ (Table 4).

Among the 3,368 orthologous gene pairs, the overall divergence in coding regions was $10.53 \%$. At non-CpG sites, the divergence was $11.37 \%$, whereas at the CpG sites, the divergence was $13.32 \%$. Apart from CpG context, the nucleotide variations in coding regions could further be classified as non-degenerative (nd) sites (any nucleotide substitutions produced amino acid changes) and four fold degenerate (4d) sites (nucleotide substitutions produced no amino acid changes). From a total of $4,713.66 \mathrm{~kb}$ of coding region sequences (CDSs), $3,009.53 \mathrm{~kb}$ were nd sites, whereas $652.4 \mathrm{~kb}$ were $4 \mathrm{~d}$ sites (Table 4). At nd sites, the overall divergence was $8.3 \%$, whereas the overall divergence at $4 \mathrm{~d}$ sites was $16.08 \%$. At nd sites, the GC content was $45.04 \%$ and the CpG content was $15.71 \%$. Among the divergence happened at nd sites, the non-CpG sites divergence between two aphid species was $9.10 \%$ and the divergence at CpG sites was $10.74 \%$. At the $4 \mathrm{~d}$ sites, the GC content was $37.20 \%$ and the CpG content was $19.88 \%$. Among the divergence happened at $4 \mathrm{~d}$ sites, the divergence was $20.25 \%$ at CpG sites while it was $17.03 \%$ at non-CpG sites. These results demonstrated that the higher percentage of divergence at $4 \mathrm{~d}$ sites compared with nd site was probably proportional to the content of $\mathrm{CpG}$ sites $(19.88 \%$ vs. $15.71 \%)$ and the rate of mutation (ts/tv). Comparison the transition and transvertion ratio of nd sites and $4 \mathrm{~d}$ sites indicated that the transvertional differences were more frequent at nd sites than at $4 \mathrm{~d}$ sites (Table 4).

Furthermore, the code usages of these 3,368 orthologous pairs between two aphid species were also analyzed by using cusp software (Alan Bleasby, ableasby@hgmp. mrc.ac.uk). The GC content of the orthologous CDSs in grain aphid was $41.66 \%$ and that in pea aphid was $39.74 \%$. The GC contents in the first, the second and the third base of triplet-codes in grain aphid were $47.78 \%, 39.38 \%$ and $37.81 \%$, respectively. However, for its counterpart, pea 
aphid, these values were $46.81 \%, 38.20 \%$ and $34.20 \%$. It was interesting to note that the first base of triplet-codes in both aphid species had highest frequency of G/C usage (or GC content) followed by second base and then the third base.

\section{Estimation of the substitution rates based on synonymous and non-synonymous analysis between grain aphid and pea aphid}

$\mathrm{Ka} / \mathrm{Ks}$ have been widely used to measure the intensity and mode of selection. $\mathrm{Ka} / \mathrm{Ks}>1$ is interpreted as a sign of positive selection whereas $\mathrm{Ka} / \mathrm{Ks}<1$ is a sign of purifying selection [15]. To identify genes undergoing positive and purifying selection, $\mathrm{Ka}$ and $\mathrm{Ks}$ substitution rates of orthologous pairs between grain and pea aphids were estimated. These 3,368 orthologous gene pairs had mean values of $\mathrm{Ka}, \mathrm{Ks}$, and $\mathrm{Ka} / \mathrm{Ks}$ of $0.016,0.085$ and 0.45 , respectively. Of these 3,368 sequence pairs, 179 orthologous gene pairs had $\mathrm{Ka} / \mathrm{Ks}$ values larger than 2,161 had $\mathrm{Ka} / \mathrm{Ks}$ values between 1 to 2 , and $229 \mathrm{had} \mathrm{Ka} / \mathrm{Ks}$ values between 0.5 and 1, and the rest 2,799 had $\mathrm{Ka} / \mathrm{Ks}$ values below 0.5 (See Additional file 4). The distribution of $\mathrm{Ka}$ and Ks was as shown in Figure 4 and the top 20 orthologs which had values of $\mathrm{Ka} / \mathrm{Ks}>1$ was as listed in Table 5.

In addition, of the 3,368 orthologous pairs, 2,088 could be annotated with KEGG terms. According to $\mathrm{Ka} / \mathrm{Ks}$ values, these 2,088 orthologs could be classified into two groups, which consisted of 186 in one group that had $\mathrm{Ka} / \mathrm{Ks}>1$ and 1,902 in another group had $\mathrm{Ka} / \mathrm{Ks}<1$. The KEGG classification of two groups which had Ka/Ks

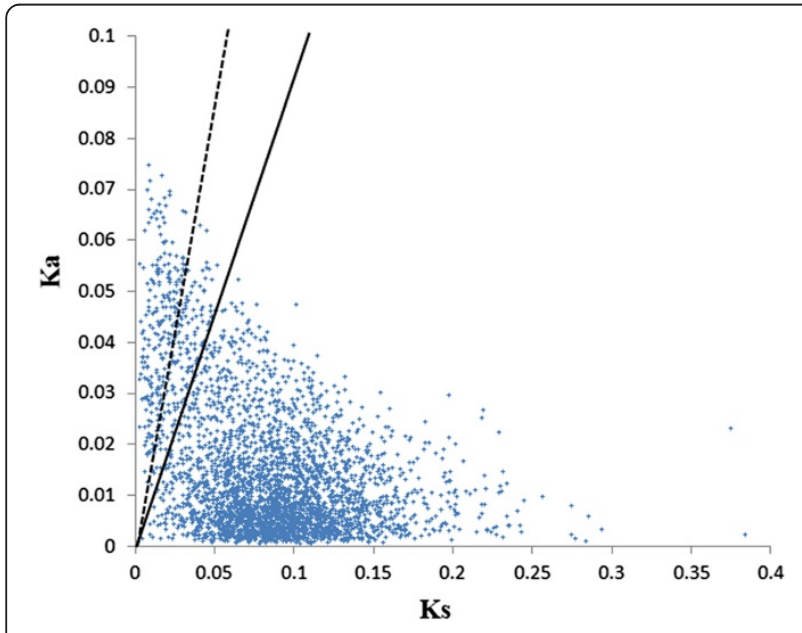

Figure 4 Distribution of Ka and Ks between orthologs in grain aphid and pea aphid. Sequences with $\mathrm{Ka} / \mathrm{Ks}>2$ fall above the dash line; while sequences with $\mathrm{Ka} / \mathrm{Ks}$ of $1-2$ fall between the solid and dash lines; Sequences with $\mathrm{Ka} / \mathrm{Ks}<1$ below the solid line. Analysis was performed using the method of Yang \& Nielsen [21]. values $<1$ and $>1$ had similar distribution patterns (See Additional file 5). To identify the KEGG pathways which had more unigenes with $\mathrm{Ka} / \mathrm{Ks}$ value larger than 1 , we compared the KEGG classifications of the two groups. The pathways such as secondary metabolisms (amino acid, polysaccharide, nucleotide, hormone, sulfur and so forth), detoxification (metabolism of xenobiotics by cytochrome P450), nucleotide-binding oligomerization domain (NOD)-like receptor signaling pathway and so on had high percentage of unigenes with value of $\mathrm{Ka} / \mathrm{Ks}>1$ (Figure 5). Given the fact that similar pathways involved were identified with the grain aphid specific unigenes (Figure 3), we proposed that these pathways might be subjected to positive selection pressure during the process of evolution and play an important role in biological variations and divergences of these two aphid species. For example, a cytochrome P450 6a2-like protein (aphid_c9724, ACYPI002699-RA), which is predicted to take part in enhancing resistance to insecticide [18], had a $\mathrm{Ka} / \mathrm{Ks}$ value larger than $1(\mathrm{Ka} / \mathrm{Ks}=3.41$, p-value of 0.049$)$. The differences in partial DNA and amino acid sequences of a cytochrome P450 6a2-like protein ortholog between grain aphid (aphid_c9724) and pea aphid (ACYPI002699-RA) were as indicated in Figure 6. Sequence alignments indicated that among the 36 nucleoid acid mutations detected, 7 were nonsynonymous substitutions leading to amino acid changes. Further $\mathrm{Ka} / \mathrm{Ks}$ analysis with sliding window length of $57 \mathrm{bp}$ and a step length of $6 \mathrm{bp}$ indicated that a locus where a nucleoid acid change of GAA to AAA which led to an amino acid change of $\mathrm{E}$ to $\mathrm{K}$ might be under positive selection during evolution $(\mathrm{Ka} / \mathrm{Ks}=1.266)$. This evidence further indicated that this cytochrome P450 6a2-like protein gene might be under strong positive selection during the evolution of the grain aphid with the observed nucleotide acids or amino acid residues subjected to these variations underlying biological divergence or host plants specialization.

\section{Discussion}

Natural selection under different ecological and agricultural environments might be the main cause of evolution and divergence among aphid species, and resulted in their biological and host plants differences. Following publication of the first pea aphid genome in February 2010, aphid biology is entering a new era focused on deciphering many of the specialized biological adaptations underlying the role of aphids as plant pests [8]. So far, only a few genes, such as mitochondrial cytochrome oxidase subunit I (mtDNA-COI), were used to study the differentiation and divergences among different populations of one or different aphid species [19]. However, the investigation of individual gene may not provide an accurate insight into the divergence underlying the biological variations and host plant adaptation of the diverse aphid species at a genome- 
Table $\mathbf{5}$ The top 20 orthologous unigenes that had Ka/Ks value larger than 1

\begin{tabular}{|c|c|c|c|}
\hline Orthology & $\mathrm{Ka} / \mathrm{Ks}$ & KEGG Annotation & Nr Annotation \\
\hline aphid_c7476 & 16.77 & - & PREDICTED: similar to hormone-sensitive lipase \\
\hline aphid_c1598 & 14.32 & - & PREDICTED: similar to E1a binding protein P400 \\
\hline aphid_c6800 & 13.51 & - & PREDICTED: similar to dusky CG9355-PA \\
\hline aphid_c28378 & 12.64 & - & PREDICTED: similar to sugar transporter, partial \\
\hline contig01882 & 12.11 & - & PREDICTED: similar to ctl transporter \\
\hline aphid_c4692 & 11.83 & $\begin{array}{l}\text { Biosynthesis of secondary } \\
\text { metabolites }\end{array}$ & PREDICTED: similar to CG9674 CG9674-PA \\
\hline aphid_c20070 & 11.58 & $\begin{array}{l}\text { Cell adhesion molecules } \\
\text { (CAMs) }\end{array}$ & Tyrosine-protein phosphatase Lar [Harpegnathos saltator] \\
\hline aphid_c9523 & 10.65 & - & PREDICTED: hypothetical protein LOC100163122, transcript variant 2 \\
\hline aphid_c381 & 10.45 & - & PREDICTED: similar to AGAP012271-PA \\
\hline aphid_c11292 & 9.95 & - & PREDICTED: similar to AGAP011393-PA \\
\hline contig00933 & 9.42 & - & $\begin{array}{l}\text { PREDICTED: similar to Zinc/iron regulated transporter-related protein } \\
3 \text { CG6898-PA }\end{array}$ \\
\hline aphid_c9186 & 8.90 & - & conserved hypothetical protein [Culex quinquefasciatus] \\
\hline aphid_c11972 & 8.78 & $\begin{array}{l}\text { Glycine, serine and } \\
\text { threonine metabolism }\end{array}$ & PREDICTED: similar to I-allo-threonine aldolase, partial \\
\hline aphid_c26862 & 8.71 & - & PREDICTED: similar to Sug CG7334-PA \\
\hline aphid_c26311 & 8.56 & - & $\begin{array}{l}\text { PREDICTED: similar to ADAM metallopeptidase with thrombospondir } \\
\text { type } 1 \text { motif, } 9 \text { preproprotein, partial }\end{array}$ \\
\hline aphid_c390 & 8.07 & - & PREDICTED: similar to Srp54 CG4602-PA \\
\hline contig03210 & 8.04 & - & PREDICTED: similar to electron-transfer-flavoprotein beta polypeptide \\
\hline aphid_c2978 & 7.83 & Purine metabolism & PREDICTED: similar to GA18461-PA \\
\hline aphid_c2071 & 7.66 & Notch signaling pathway & PREDICTED: similar to nuclear receptor co-repressor 1 \\
\hline aphid_c9176 & 7.36 & $\begin{array}{l}\text { Arachidonic acid } \\
\text { metabolism }\end{array}$ & PREDICTED: similar to AGAP010241-PA [Acyrthosiphon pisum] \\
\hline
\end{tabular}

wide scale. Genome information for additional aphid species needs to be obtained to perform the high-resolution comparative genomic and evolutionary analyses [8]. Comparison between complete genome sequence of pea aphid (as a reference gene set) and expressed sequence tag (EST) data from three other species, green peach-potato aphid (Myzus persicae), cotton aphid (Aphis gossypii) and Toxoptera citricida suggested that a number of the genes evolved fast (high ratio of $\mathrm{Ka} / \mathrm{Ks}$ ), including many genes shared by aphids but with no hit in universal proteins (Uniprot) [13]. Furthermore, comparisons of the genome sequence of pea aphid with ESTs of 8 other aphid species were also performed to verify whether the accumulation of deleterious mutations is the reason for loss of sexual reproduction [14]. Yet, no similar works on grain aphid or the transcriptome data of grain aphid were documented so far.

A more robust picture of genomic divergence among these major agricultural important aphid species may be obtained by examining the transcriptome that have been selected in an unbiased way without prior interest in their biological functions or evolutionary histories [20]. Although the transcriptome data depend on the environment of aphids sampled as well as how the genotype has evolved, the transcriptome represents a sample of the spatiotemporally-expressed genome and can be used as an entry into the genome divergence analysis [20]. In this study, we analyzed the divergence of transcriptome sequence between the two aphid species, and similar genes/pathways were identified to be involved in the divergence of two aphid species through both comparative transcriptomic analysis and $\mathrm{Ka} / \mathrm{Ks}$ analysis. Comparing with mRNA sequences of pea aphid, 4,857 unigenes were regarded to be specifically expressed in grain aphid under the described rearing conditions (Methods), among which could be annotated by KEGG terms, most of them were involved in secondary metabolism pathways, amino acid metabolism, purine and pyrimidine metabolism and detoxification or insecticide resistance (Figure 3). In addition, to estimate the extent to which selection affects proteincoding sequences, the ratios of $\mathrm{Ka} / \mathrm{Ks}$ were estimated. $\mathrm{Ka} /$ $\mathrm{Ks}$ ratio is a good indicator of selective pressure and has been used to identify protein coding sequences under positive and purifying selection [21]. $\mathrm{Ka} / \mathrm{Ks}$ analysis between grain aphid and pea aphid showed that 340 orthologs were identified to have $\mathrm{Ka} / \mathrm{Ks}>1$, which was interpreted as a 


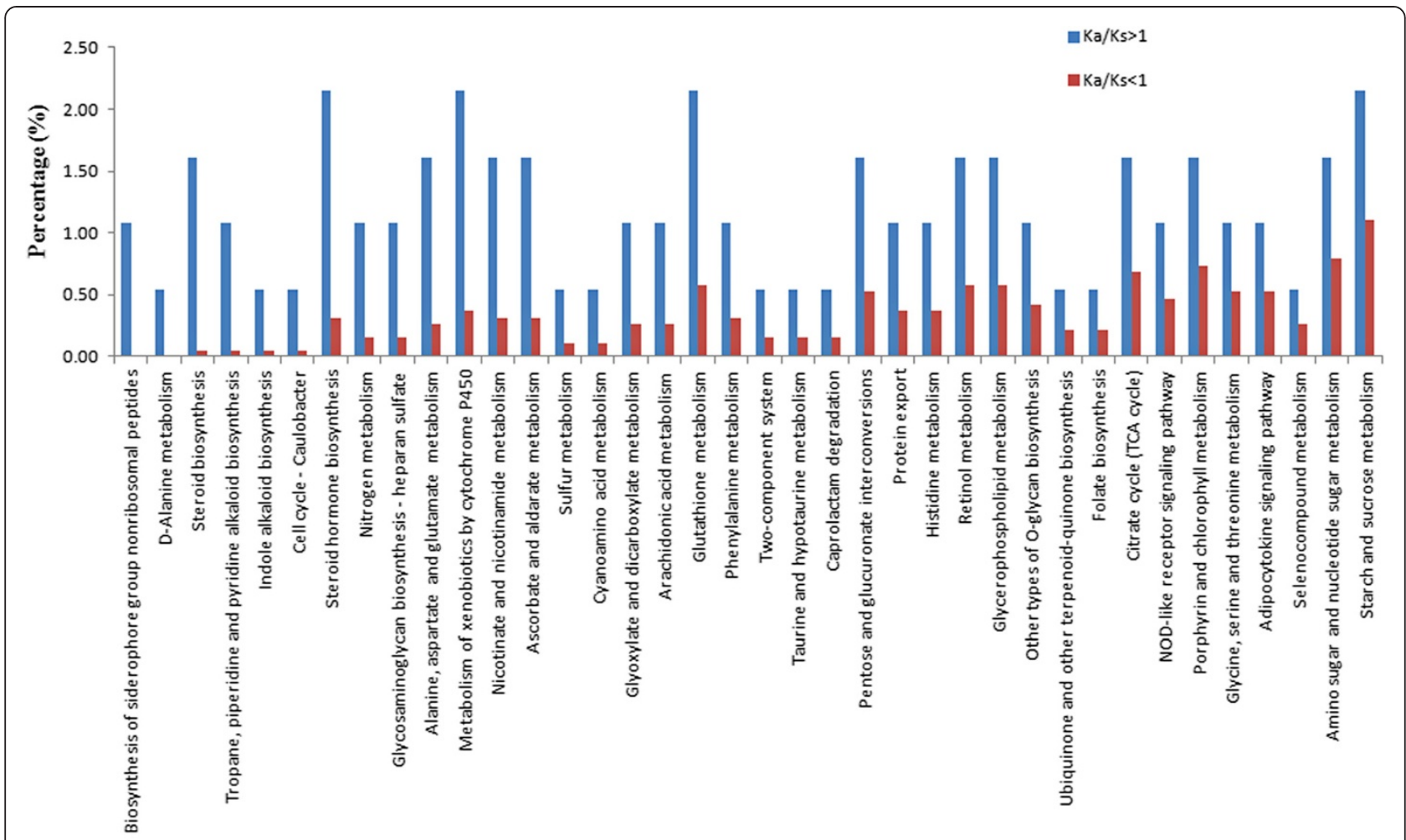

KEGG pathway terms

Figure $\mathbf{5}$ Comparison of the KEGG pathways of orthologs with ratios of $\mathrm{Ka} / \mathrm{Ks}>\mathbf{1}$ and $\mathrm{Ka} / \mathrm{Ks}<\mathbf{1}$. Unigenes involved in pathways such as secondary metabolisms (amino acid, polysaccharide, nucleotide, hormone, sulfur and so forth), detoxification (metabolism of xenobiotics by cytochrome P450), signaling (NOD-like receptor signaling pathway) and so on had higher Ka/Ks values due to positive selection.

sign of positive selection. Many of these genes were involved in KEGG pathways such as amino acid biosynthesis and other biomolecular synthesis and metabolism, polysaccharide metabolism, insecticide resistance and detoxification, and NOD-like receptor signaling pathway (Figure 5). Given that similar involved pathways were identified with the grain aphid specific unigenes, such as secondary metabolism, purine and pyrimidine metabolism and amino metabolism, detoxification and insecticide resistance, these lines of evidences suggested that the above mentioned biological processes and/or pathways were under strongly positive selection and might play important roles in divergence of biological variation and host plants adaptation during the aphid evolution. Similar processes were also identified to be involved in the divergence between two invasive whitefly cryptic species [22].

In addition, a number of divergent sequences within the orthologous gene pairs might also contribute to the biological differences between the two aphid species. The gene divergence at the 5'UTR (21.29\%) was more obvious than that of CDS (10.53\%) and 3'UTR regions (18.96\%). The ratio of ts/tv can indicate the mechanism of evolution [16]. The frequencies of ts were higher than that of tv both in 5'UTR and 3' UTR region. However, in the coding region, this frequency was slightly lower (Table 4). This result further indicated that the sequence divergences of these two aphid species in un-coding regions of orthologous gene pairs were mainly caused by transitional difference whereas that of the coding regions was mainly due to the transversional variations, especially at the nd sites in which the change of every base of codon would cause the variation of amino acid sequence.

Although the transcriptome of grain aphid generated in our lab and used in this study might have biases due to the fact that the presence of the transcriptome profile depends on the conditions under which aphids were reared and the different developmental stages of aphids, it did not indeed affect the grain aphid specific sequences and orthologous pairs identified by ETE software using a species overlap score (SOS) of 0.0 because of the following reasons. Firstly, we used aphids of mixed life stages to make the library in order to maximize the likelihood of having a whole set of transcripts under the described rearing condition in this study. Secondly, analysis of the transcriptomic 


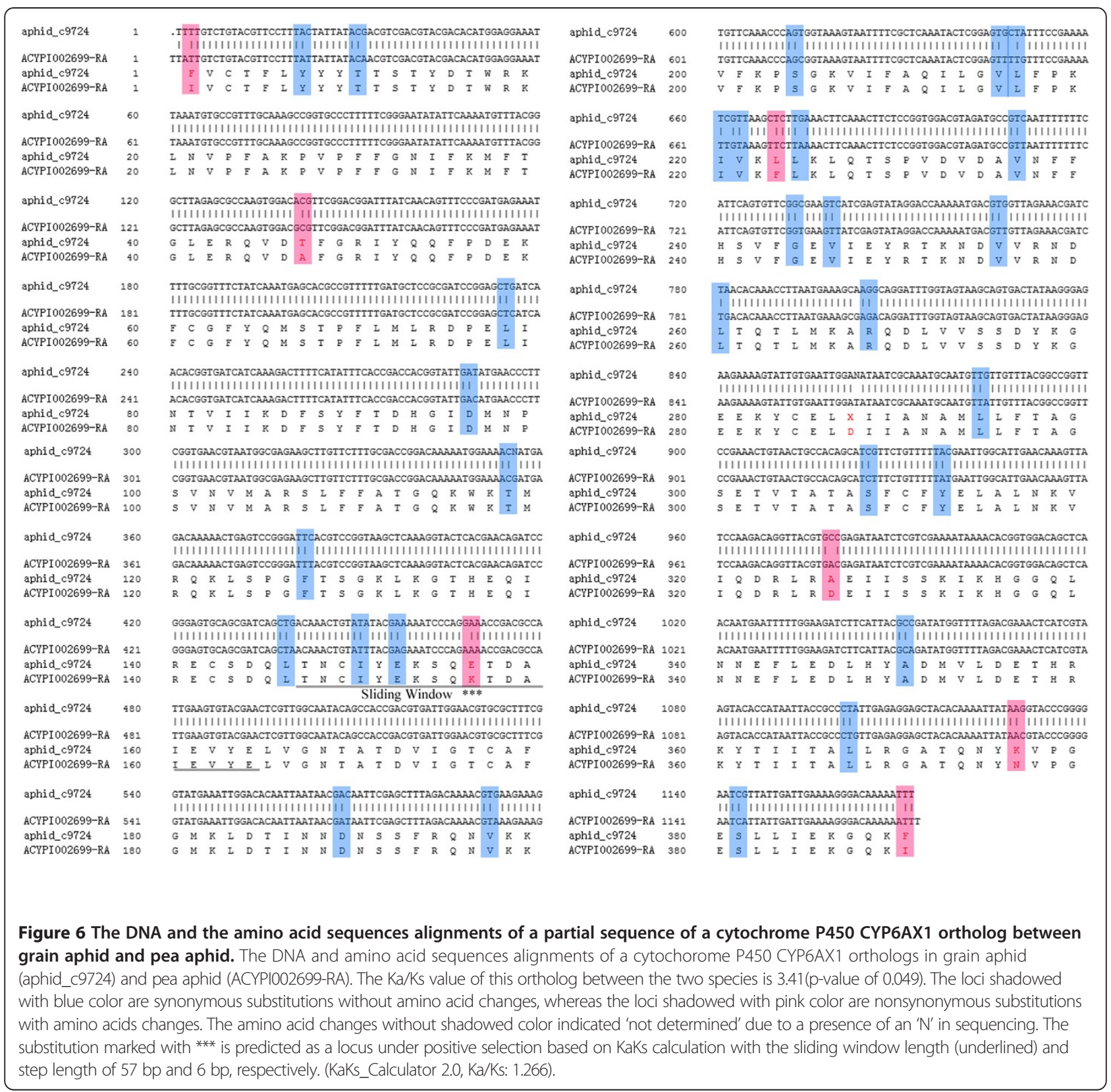

divergence under the same rearing conditions such as in an artificial diet assay may also not appropriate because some genes involved in host plants localization and aphidplant interaction may not expressed at all. At last, the generated transcriptome was assembled by using the genome sequences of pea aphid as a reference set. Based on this, grain aphid specific sequences and sequence divergences between the orthologous pairs of these aphid species was analyzed and divergences in the orthologous pairs in the coding, 5'UTR and 3' UTR regions were explored with ETE with SOS of 0.0. ETE algorithm uses the level of species overlap between the two branches of a given node to define a duplication $(\mathrm{SOS}>0.0)$ or a speciation $(\mathrm{SOS}=0.0)$ and this has been successfully applied in comparison of gene repertoires and patterns of evolutionary rates in eight aphid species that differ by reproductive mode $[14,23]$. And then 186 orthologs which were predicted to be involved in secondary metabolism, xenobiotic metabolisms and etc. were identified to have evolved in response to positive selection based on Ka and Ks analyses. However, we can't expect to solve all the problems with one single silver bullet, the revealed genetic divergence between grain aphid and pea aphid remains to be appropriately interpreted in light of their biological studies in the near future.

Nevertheless, despite the high sequence homology between grain aphid and pea aphid and its closer relationship, 
both the transcriptome divergences and the $\mathrm{Ka} / \mathrm{Ks}$ analyses demonstrated that grain aphid and pea aphid have diverged substantially. And pathways such as the amino acids and other biomolecular synthesis and metabolism, polysaccharide and saccharide metabolism, insecticide resistance and detoxification, might have been subjected to strongly positive selection and play important roles in biological divergences of these two agriculturally important aphid species.

\section{Conclusions}

Comparative analysis between this set of transcriptome of grain aphid generated in our lab by Roche 454 GSFLX pyrosequencing and mRNA sequences of pea aphid available in the public databases revealed both grain aphid specific sequences and divergent orthologous sequences. And a couple of orthologous genes and/or pathways were identified to have evolved under positive selection pressure which might play important roles in the divergences of grain aphid and pea aphid. To our knowledge, this is the first attempt to study the transcriptome divergence of these two agriculturally important pest species. Our results will provide valuable resources for post-genomic studies of fundamental biological questions in both grain and pea aphids, and investigation of the molecular mechanisms underlying the evolution and divergence of these two destructive aphid species.

\section{Methods}

\section{Materials}

Apterous adult grain aphids derived from a single clonal lineage reared on wheat (Triticum aestivum L cv Kenong 199) seedlings were placed in cages for $24 \mathrm{~h}$ to produce nymphs. Ten neonate nymphs produced in the $24 \mathrm{~h}$ period were transferred to fresh wheat plants. 12 days later, the offspring of these aphids, were selected and subjected to transcriptomic sequencing experiment. Then, 300 grain aphids at different development stages, for example, 60 first instars, 60 second instars, 60 third instars, 60 fourth instars and 60 adults were collected from wheat seedlings with a brush and immediately frozen in liquid nitrogen, and stored at $-80^{\circ} \mathrm{C}$ for RNA extraction.

\section{RNA sequencing}

Total RNA was isolated using a Qiagen RNA Extraction kit according to the manufacturer's instructions (New England BioLabs). It was treated with RNase-free DNase I for $30 \mathrm{~min}$ at $37^{\circ} \mathrm{C}$ to remove residual DNA, and mRNA was isolated from DNA-free total RNA using a PolyA Tract Kit (Promega, USA) according to the manufacturer's instructions. In total $500 \mathrm{ng}$ of mRNA isolated from the total RNA, was fragmented by $\mathrm{ZnCl}_{2}$ solution, and then purified and condensed with RNeasy MinElute RNA Cleaning up Kit (Qiagen, Germany). The fragmented RNA was eluted from the membrane of the spin tube with $10 \mu$ l of RNase- free water, and reverse transcribed into first strand cDNA with a random primer and AMV reverse transcriptase. The second strand of cDNA was synthesized by DNA polymerase $\mathrm{I}, \mathrm{RNase} \mathrm{H}$ and ligase enzyme mixtures. After being blunted and appended with an Adenine base overhang at the 3 ' end, the double-stranded cDNA were ligated with GS-FLX sequencing adaptors. The fragments shorter than $500 \mathrm{bp}$ were removed by Ampure Beads according to the manufacturer's instructions. We used a fluorescence photometer to titer the library and a High Sensitivity DNA Analysis Chip kit to verify the length distribution of the cDNA library.

The small volume (SV) and large volume (LV) emPCRs were carried out according to emPCR kit manual. The enriched beads were counted with a Beckman Coulter Z1, and 2,000,000 beads were loaded into each of 1/2 PicoPlate region. After the beads were loaded into Pico-Plate, the sequencing reaction was carried out with the parameters of two regions, XL70Li and 200 cycles in a GS-FLX sequencing machine. The image processing and base calling were processed on a 454 data process cluster, automatically.

\section{Data assembly and analysis}

All the raw sequences were then processed to remove low quality and adaptor sequences by using programs such as TagDust [24], LUCY [25] and SeqClean [26] with default parameters. The resulting sequences were then screened against the NCBI UniVec database (http:// www.ncbi.nlm.nih.gov/VecScreen/UniVec.html, version 20101122) to remove possible vector sequence contamination. Sequences shorter than 50 bp were discarded. The cleaned data were denovo assembled using Mira 3 with the parameter of "denovo", "accurate" and "est" (mira - project $=$ aphid - job $=$ denovo, est, accurate,454fasta $=$ aphid.454.fasta 454_SETTINGS-CL:qc = no.) [27]. The resulting contigs and singletons that were more than $100 \mathrm{bp}$ were retained. To overcome the under-assembled problem of Mira 3 assembler and enhance the quality of assembling, the unigenes were reassembled using gsAssembler v2.3 with an identity parameter of 95\% (Roche NimbleGen, Inc., Madison, WI, USA). To eliminate trace contamination of Buchnera aphidicola transcripts, which may be introduced due to the miss-paired sequences to oligo( $\mathrm{T})_{20}$ probe using PolyA Tract Kit during mRNA separation, the re-assembled data were filtered by searching against genome sequence of Buchnera aphidicola (GenBank no: BA000003.2) using BLAST with a cutoff E-value of 1E-10 [28]. BLAST against public available databases was performed to annotate the functions of these unigenes by using E-value cutoffs of $1 \mathrm{E}-10,1 \mathrm{E}-5$ and $1 \mathrm{E}-5$ for $\mathrm{Nt}, \mathrm{Nr}$ and Swiss-Prot, respectively [29,30]. The databases used for functional annotation included $\mathrm{Nr}$ (http://www.ncbi.nlm. nih.gov; version 20101011), Nt (http://www.ncbi.nlm.nih.gov, 
version 20101011), and Swiss-Prot (http://www.ebi.ac.uk/ uniprot, version 20090819). Moreover, functional classifications were also conducted including Gene Ontology (GO, http://geneontology.org/), KOG (http://www.ncbi.nlm.nih. gov/COG/) and the KEGG pathway (http://www.genome. jp/kegg). GO annotations were conducted by searching against the $\mathrm{Nr}$ database using Blast2GO (E-value of 1E-6) [31]. EGO and a custom script were used for assignment of each GO ID to the related ontology entries [32]. KOG and KEGG classifications were performed using BLAST (E-value of 1E-6). ORF analyses were performed by ORF finder (http://www.ncbi.nlm.nih.gov/gorf/gorf.html).

\section{Synonymous and non-synonymous analysis}

The CDS of each sequences of grain aphid were predicted by ORF finder, and then extracted according to the predicted results by a personal perl script written for this purpose. Orthologous relationships between grain aphid and pea aphid genes were conducted by using ETE [23]. We performed a BLAST against predicted genes in pea aphid (BlASTn, a cut-off E-value of 1E-10) for each of the unigene of grain aphid. The sequences aligned with a continuous region longer than $50 \%$ of the query sequence were retained and aligned using Muscle 3.8 [33]. All columns in the sequences alignments with gaps were removed using trimAL [34] (http://trimal.cgenomics.org/). Then, the orthologous gene pairs between two aphid species were inferred using the species overlap algorithm described in ETE by using a species overlap score (SOS) of 0.0 [23]. At the same time, we checked the alignments manually to ensure these orthologs were true ones rather than the artifacts of EST assembly.

Coding sequences (CDS) of the orthologous genes were determined using BLASTx software and the CDS sequence of each unigene was extracted using a perl script written for this purpose. The perl script for this analysis was as indicated in Additional file 6. Orthologs were matched by MegaBLAST and orthologous pairs longer than $150 \mathrm{bp}$ were retained. Then, substitution rates of these orthologous genes between grain and pea aphid were estimated separately for synonymous (Ks) and non-synonymous sites (Ka) using an approximate method implemented in the software KaKs Calculator Version 1.2 [15]. Pair-wise approximate analysis were performed using the YN method [21]. As the sequencing errors were distributed among synonymous and nonsynonymous sites at equal frequencies, they were not expected to influence the results of analyses [35].

\section{The GC contents and CpG analysis}

GC contents of unigenes, 5'UTRs, 3' UTRs and CDSs were generated by a perl script written for this purpose (See Additional file 6). The sequence divergence was calculated by dividing the number of substitutions by the number of base pairs compared. The divergence was determined for the contexts of non-degenerate (nd), fourfold degenerate (4d), CpG and non-CpG [36]. The code usages of orthologous gene pairs were analyzed using cusp software (Alan Bleasby, ableasby@hgmp.mrc.ac.uk).

\section{Additional files} Additional file 1: The flow chart of the assembly of the grain aphid
transcriptome data.

Additional file 2: Annotations and the classifications of the assembled unigenes of grain aphid.

Additional file 3: The KEGG pathway classifications grain aphid specific unigenes.

Additional file 4: The $\mathrm{Ka}, \mathrm{Ks}$ and $\mathrm{Ka} / \mathrm{Ks}$ values of the orthologous gene pairs between grain aphid and pea aphid.

Additional file 5: The KEGG pathway classifications of orthologs with the value of $\mathrm{Ka} / \mathrm{Ks}>1$ and $\mathrm{Ka} / \mathrm{Ks}<1$ between grain aphid and pea aphid.

Additional file 6: The perl scripts used in this study.

\section{Abbreviations}

BLAST: Basic Local Alignment Search Tool; CDS: Coding Sequence; ESTs: Expressed Sequence Tags; GO: Gene Ontology; KEGG: Kyoto Encyclopedia of Genes and Genomes; KOG: Eukaryotic Orthologous Groups of Proteins; Nr: NCBI Non-redundant Protein Database; Nt: NCBI Nucleotide Acid Database; qPCR: Quantitative Polymerase Chain Reaction; RPKM: Read Per Kb Per Million Bases; RT-PCR: Reverse-Transcript Polymerase Chain Reaction; Uniprot: Universal Protein.

\section{Competing interests}

The authors declare that they have no competing interests.

\section{Authors' contributions}

LQX conceived and planned the project. DHW carried out most of the experiments. QL and DHW analyzed the transcriptome data. DHW and LQX wrote the manuscript. HDJ and TB revised the manuscript. Most of the experiments were carried out in the Molecular Laboratory of Beijing Autolab Biotechnology Co. Ltd. All authors read and approved the final manuscript.

\section{Acknowledgements}

The authors sincerely thank Prof. Linda M. Field from Rothamsted Research, UK for her critical review of this manuscript. This work was supported by grants from the Research Initiative on Transgenic Plants from the Ministry of Agriculture of China (grant no. 2014ZX0800201B), Natural Science Foundation of China (grant no.31171618, 31371702) and the Chinese State Key Laboratory for Biology of Plant Diseases and Insects (grant no. SKLOF201307). Rothamsted Research receives grant-aided support from the Biotechnology and Biological Sciences Research Council (BBSRC) of the UK.

\section{Author details}

${ }^{1}$ Institute of Crop Sciences/The National Key Facility for Crop Gene Resource and Genetic Improvement, Chinese Academy of Agricultural Sciences (CAAS), 12 Zhongguanchun South Street, Beijing 10081, China. ${ }^{2}$ Beijing Autolab Biotechnology Co. Ltd, Eastern Campus, Beijing Jiaotong University, Beijing 100081, China. ${ }^{3}$ The Medical College of Wenzhou, Wenzhou 325032, China. ${ }^{4}$ Rothamsted Research, Harpenden, Hertfordshire AL5 2JQ, UK.

Received: 16 December 2013 Accepted: 6 November 2014 Published: 25 November 2014

\section{References}

1. International Aphid Genomics Consortium: Genome sequence of the pea aphid Acyrthosiphon pisum. PLOS Biol 2010, 8(2):e1000313.

2. Morrison WP, Peairs FB: Response Model Concept and Economic Impact. In Response Model for an Introduced Pest: The Russian Wheat Aphid. Edited 
by Quisenberry SS, Peairs FB. Lanham: MD: Entomological Society of America; 1998:1-11.

3. Awmack CS, Harrington R: Elevated $\mathrm{CO} 2$ affects the interactions between aphid pests and host plant flowering. Agric Forest Entomol 2001, 2:57-61.

4. Zhang YJ, Jiang YY, Feng $X D$, Xia B, Zeng J, Liu Y: Occurring trends of major crop pests in China in 2009. China Plant Prot 2009, 29:33-36.

5. Stoger ES, Williams S, Christou P, Down RE, Gatehouse JA: Expression of the insecticidal lectin from snowdrop (Galanthus nivalis agglutinin; GNA) in transgenic wheat plants: effects on predation by the grain aphid Sitobion avenae. Mol Breed 1999, 5:65-73.

6. Yu XD, Pickett J, Ma YZ, Bruce T, Napier J, Jones HD, Xia LQ: Metabolic engineering of plant-derived (E)- $\beta$-farnesene synthase genes for a novel type of aphid-resistant genetically-modified crop plants. J Integr Plant Biol 2012, 54(5):282-299.

7. Legeai F, Shigenobu S, Gauthier JP, Colbourne J, Rispe C, Collin O, Richards S, Wilson AC, Murphy T, Tagu D: AphidBase: a centralized bioinformatic resource for annotation of the pea aphid genome. Insect Mol Biol 2010, 19:5-12.

8. International Aphid Genomics Consortium: Aphid White Paper II: Proposal to Complete Development of the Aphid Model. 2010. IAGC-White Paper II-FINAL VERSION-September 13th $2010 \mathrm{http}: / /$ www.academia.edu/ 973082/Aphid_White_Paper_II_Proposal_to_Complete_Development_ of the_Aphid_Model.

9. Zhang M, Zhou Y, Wang H, Jones HD, Gao Q, Wang DH, Ma YZ, Xia LQ: Identifying potential RNAi targets in grain aphid (Sitobion avenae F.) based on transcriptome profiling of its alimentary canal after feeding on wheat plants. BMC Genomics 2013, 14:560.

10. Wang Z, Gerstein M, Snyder M: RNA-Seq: a revolutionary tool for transcriptomics. Nat Rev Genet 2009, 10:57-63.

11. Kahvejian A, Quackenbush J, Thompson JF: What would you do if you could sequence everything? Nat Biotechnol 2008, 26:1125-1133.

12. Morrissy AS, Morin RD, Delaney A, Zeng T, McDonald H, Jones S, Zhao Y, Hirst M, Marra MA: Next-generation tag sequencing for cancer gene expression profiling. Genome Res 2009, 19:1825-1835.

13. Ollivier M, Legeai F, Rispe C: Comparative analysis of the Acyrthosiphon pisum genome and expressed sequence tag-based gene sets from other aphid species. Insect Mol Biol 2010, 19(Suppl. 2):33-45.

14. Ollivier M, Gabaldón T, Poulain J, Gavory F, Leterme N, Gauthier JP, Legeai F, Tagu D, Simon JC, Rispe C: Comparison of gene repertoires and patterns of evolutionary rates in eight aphid species that differ by reproductive mode. Genome Biol Evol 2012, 4(2):155-167.

15. Zhang Z, Li J, Zhao XQ, Wang J, Wong GK, Yu J: KaKs_Calculator: calculating Ka and Ks through model selection and model averaging. Genomics Proteomics Bioinformatics 2006, 4:259-263.

16. Yang Z, Yoder AD: Estimation of the transition/transversion rate bias and species sampling. J Mol Evol 1999, 48:274-283

17. Swanson WJ, Clark AG, Waldrip-Dail HM, Wolfner MF, Aquadro CF: Evolutionary EST analysis identifies rapidly evolving male reproductive proteins in Drosophila. Proc Natl Acad Sci U S A 2001, 98:7375-7379.

18. Brun A, Cuany A, Le Mouel T, Berge J, Amichot M: Inducibility of the Drosophila melanogaster cytochrome P450 gene, CYP6A2, by phenobarbital in insecticide susceptible or resistant strains. Insect Biochem Mol Biol 1996, 26(7):697-703.

19. Pang YW, Ma EB, Ren ZM: Taxonomic and phylogenetic status of Rhus gall aphid Melaphis rhois (Homoptera: Pemphigidae) from North America based on morphological characters and mtDNA COI gene sequence. Acta Entomol Sin 2011, 54:575-581.

20. Elmer KR, Fan S, Gunter HM, Jones JC, Boekhoff S, Kuraku S, Meyer A: Rapid evolution and selection inferred from the transcriptomes of sympatric crater lake cichlid fishes. Mol Ecol 2010, 19:197-211.

21. Yang Z, Nielsen R: Estimating synonymous and nonsynonymous substitution rates under realistic evolutionary models. Mol Bio Evol 2000 17:32-43.

22. Wang XW, Luan JB, Li JM, Su YL, Xia J, Liu SS: Transcriptome analysis and comparison reveal divergence between two invasive whitefly cryptic species. BMC Genomics 2011, 12:458.

23. Huerta-Cepas J, Dopazo H, Dopazo J, Gabaldon T: The human phylome. Genome Biol Evol 2007, 8(6):R109.

24. Lassmann T, Hayashizaki Y, Daub CO: TagDust-a program to eliminate artifacts from next generation sequencing data. Bioinformatics 2009 $25: 2839-2840$
25. Chou HH, Holmes MH: DNA sequence quality trimming and vector removal. Bioinformatics 2001, 17:1093-1104.

26. Chen YA, Lin CC, Wang CD, Wu HB, Hwang PI: An optimized procedure greatly improves EST vector contamination removal. BMC Genomics 2007, 8:416.

27. Chevreux B, Pfisterer T, Drescher B, Driesel AJ, Müller WE, Wetter T, Suhai S: Using the miraEST assembler for reliable and automated mRNA transcript assembly and SNP detection in sequenced ESTs. Genome Res 2004, 14:1147-1159

28. Tamas I, Klasso L, Canback B, Naslund AK, Eriksson AS, Wernegreen Jj: 50 million years of genomic stasis in endosymbiotic bacteria. Science 2002, 296:2376-2379.

29. Karatolos N, Pauchet Y, Wilkinson P, Chauhan R, Denholm I, Gorman K, Nelson DR, Bass C, french-Constant RH, Williamson MS: Pyrosequencing the transcriptome of the greenhouse whitefly. Trialeurodes vaporariorum reveals multiple transcripts encoding insecticide targets and detoxifying enzymes. BMC Genomics 2011, 12:56

30. Hoffman JI, Thorne MAS, Trathan PN, Forcada J: Transcriptome of the dead: characterisation of immune genes and marker development from necropsy samples in a free-ranging marine mammal. BMC Genomics 2013, 14:52.

31. Conesa A, Gotz S, Garcia-Gomez JM, Terol J, Talon M, Robles M: Blast2GO: a universal tool for annotation, visualization and analysis in functional genomics research. Bioinformatics 2005, 21:3674-3676.

32. Ye J, Fang L, Zheng H, Zhang Y, Chen J, Zhang Z, Wang J, Li S, Li R, Bolund L: EGO: a web tool for plotting GO annotations. Nucleic Acids Res 2006, 34:293-297.

33. Edgar RC: MUSCLE: multiple sequence alignment with high accuracy and high throughput. Nucleic Acids Res 2004, 32(5):1792-1797.

34. Capella-Gutiérrez Silla-Martínez MJ, Gabaldón T: TrimAl: a tool for automated alignment trimming in large-scale phylogenetic analyses. Bioinformatics 2009, 25(15):1972-1973.

35. Tiffin P, Hahn MW: Coding sequence divergence between two closely related plant species: Arabidopsis thaliana and Brassica rapa ssp. pekinensis. J Mol Evol 2002, 54:746-753.

36. Hellmann I, Zollner S, Enard W, Ebersberger I, Nickel B, Paabo S: Selection on human genes as revealed by comparisons to chimpanzee cDNA. Genome Res 2003, 13:831-837.

doi:10.1186/1471-2164-15-1023

Cite this article as: Wang et al: Comparative transcriptomic analyses revealed divergences of two agriculturally important aphid species. BMC Genomics 2014 15:1023.

\section{Submit your next manuscript to BioMed Central and take full advantage of:}

- Convenient online submission

- Thorough peer review

- No space constraints or color figure charges

- Immediate publication on acceptance

- Inclusion in PubMed, CAS, Scopus and Google Scholar

- Research which is freely available for redistribution 